\title{
Kasr Alainy simplified conservative uterine surgery for abnormally invasive placenta (AIP): surgical technique
}

\author{
abdalla Mousa ${ }^{1}$, Ahmed Maged ${ }^{1}$, Islam Elkhateb ${ }^{2}$, Hassan Gaafar ${ }^{1}$, Tommy Mousa ${ }^{3}$, \\ Mohamed Abdel-Fattah ${ }^{4}$, Ahmed Mourad ${ }^{1}$, Moutaz Elsherbini $^{1}$, and Rasha Elkomy ${ }^{1}$ \\ ${ }^{1}$ Cairo University Kasr Alainy Faculty of Medicine \\ ${ }^{2}$ Newgiza University \\ ${ }^{3}$ University Hospitals of Leicester NHS Trust \\ ${ }^{4}$ University of Aberdeen Dugald Baird Centre for Research on Women's Health
}

October 15, 2020

\begin{abstract}
The incidence of placenta accreta has risen significantly owing to global surge in caesarian section rates. It is associated with significant morbidities and mortality and is usually managed with caesarean hysterectomy $(\mathrm{CsH})$. Uterine preserving conservative surgeries have advantages over $\mathrm{CsH}$ and leaving placenta in-situ techniques. We present a new conservative surgery technique that was applied on 20 patients with abnormally invasive placenta. It was successful in $90 \%$ of cases for preservation of the uterus. It was associated with relatively low blood loss and morbidity outcomes. Our simplified easy to learn technique can be useful especially for countries with limited resources.
\end{abstract}

\section{Hosted file}

Mousa_October_2020_manuscript.pdf available at https://authorea.com/users/367366/articles/ 486827-kasr-alainy-simplified-conservative-uterine-surgery-for-abnormally-invasiveplacenta-aip-surgical-technique

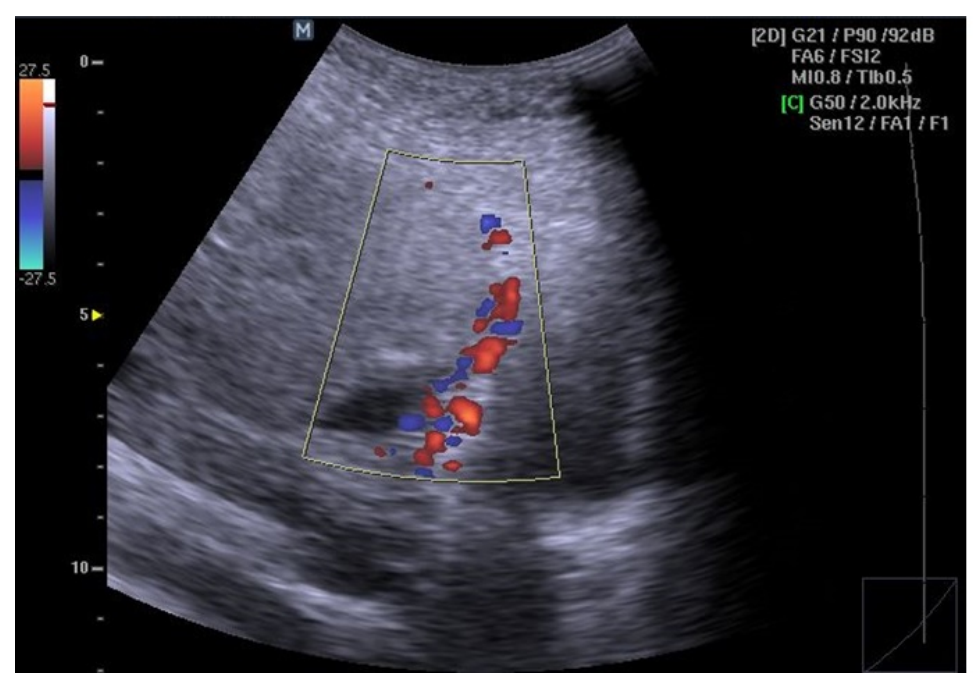



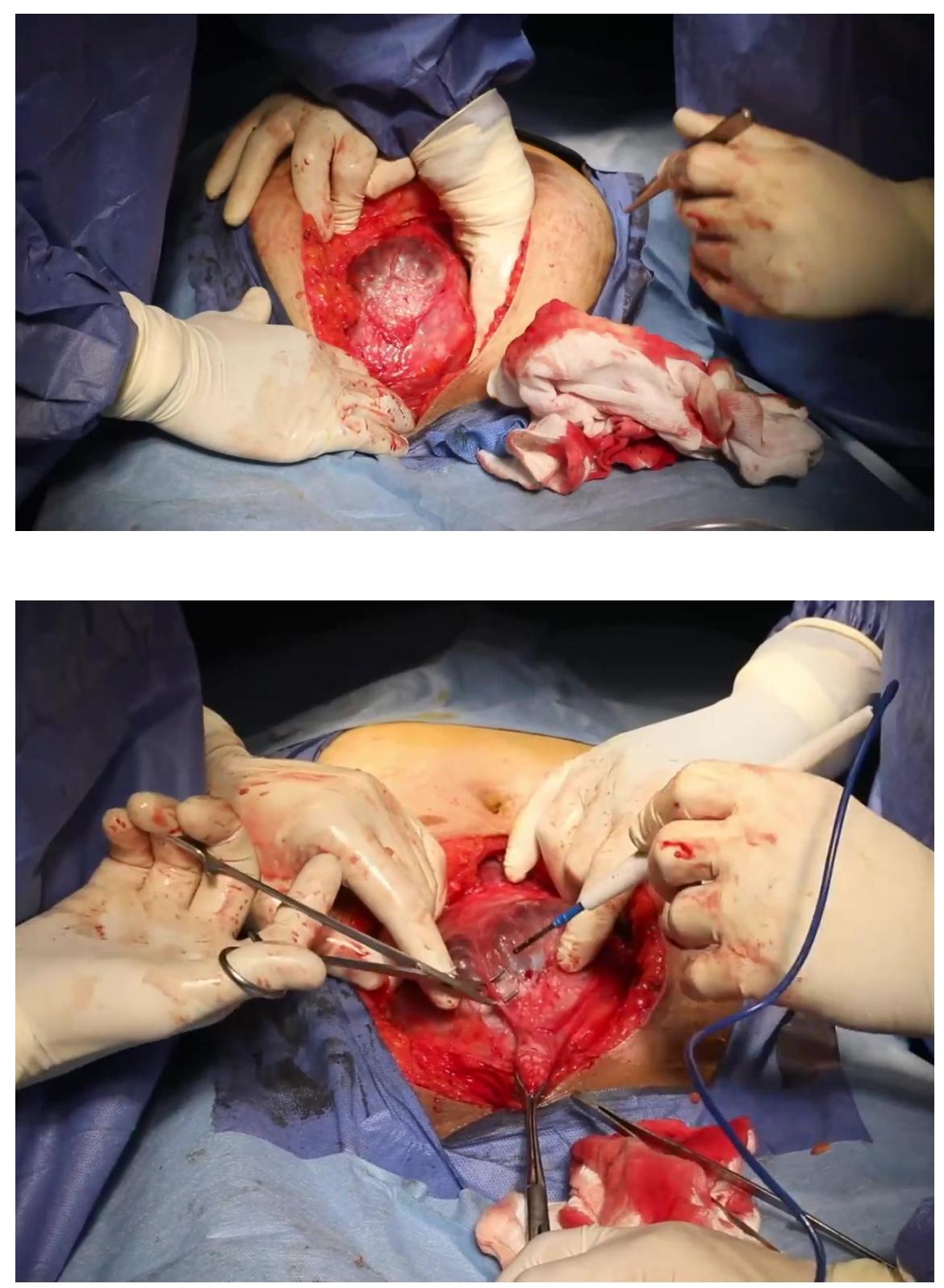

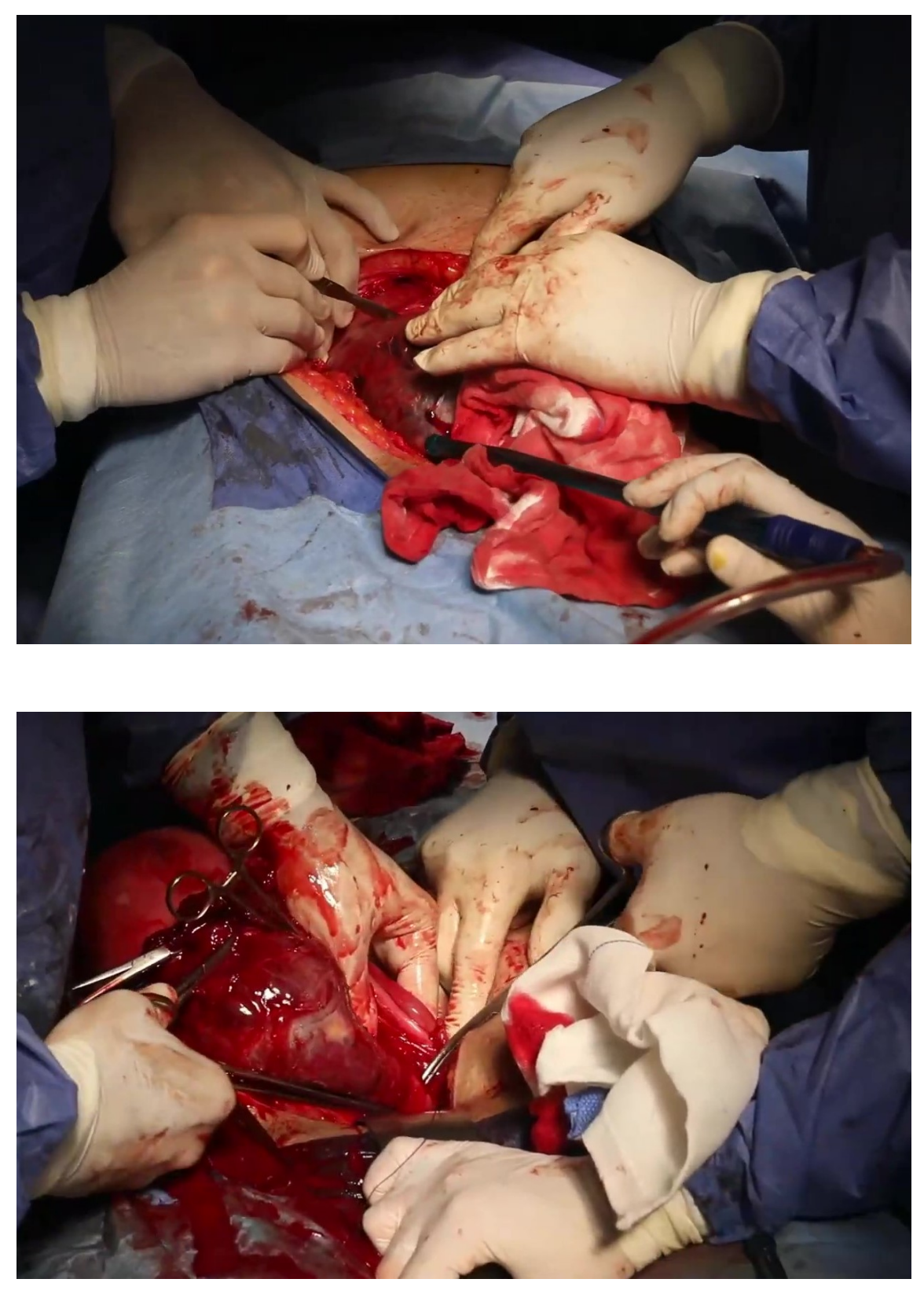

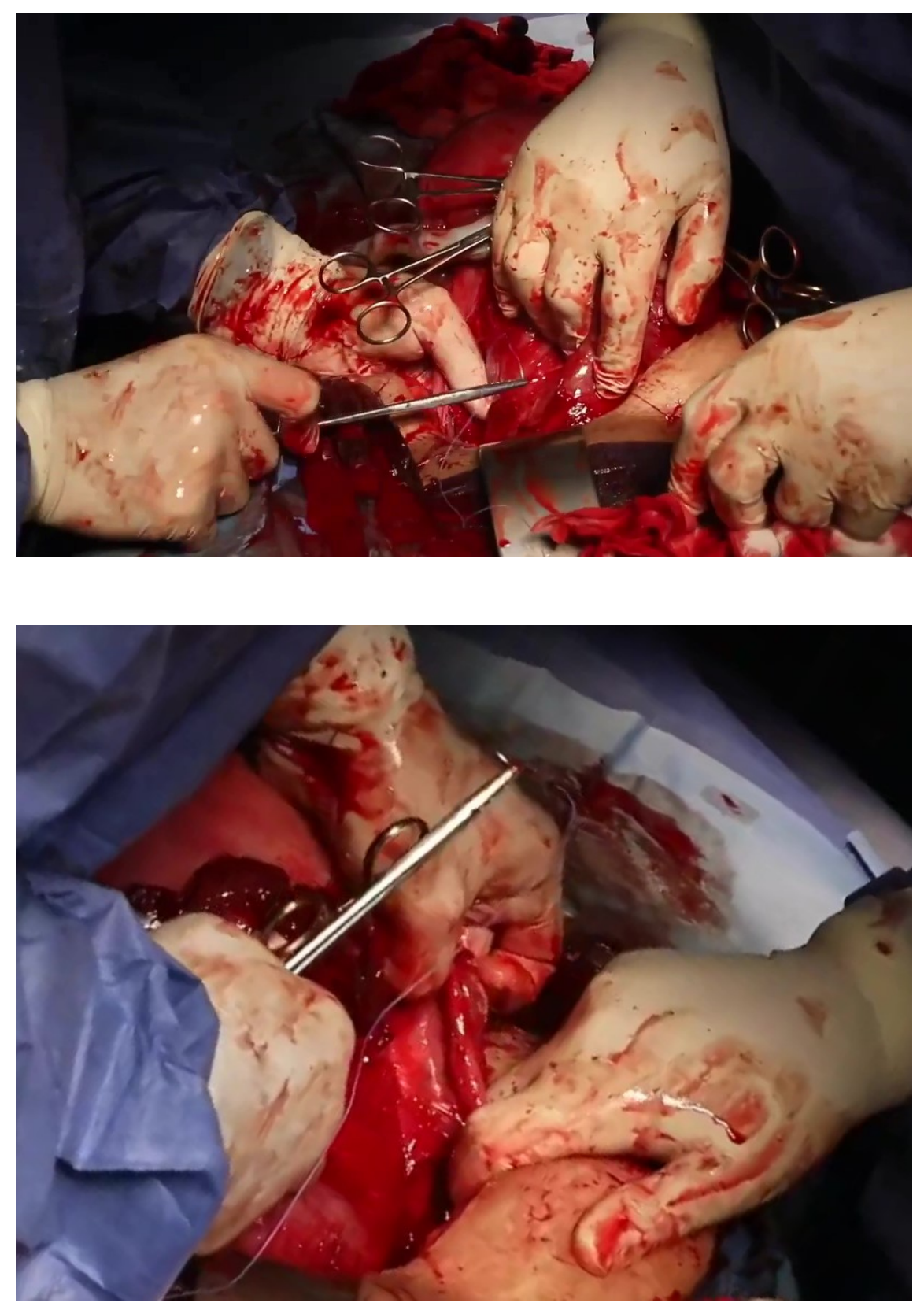

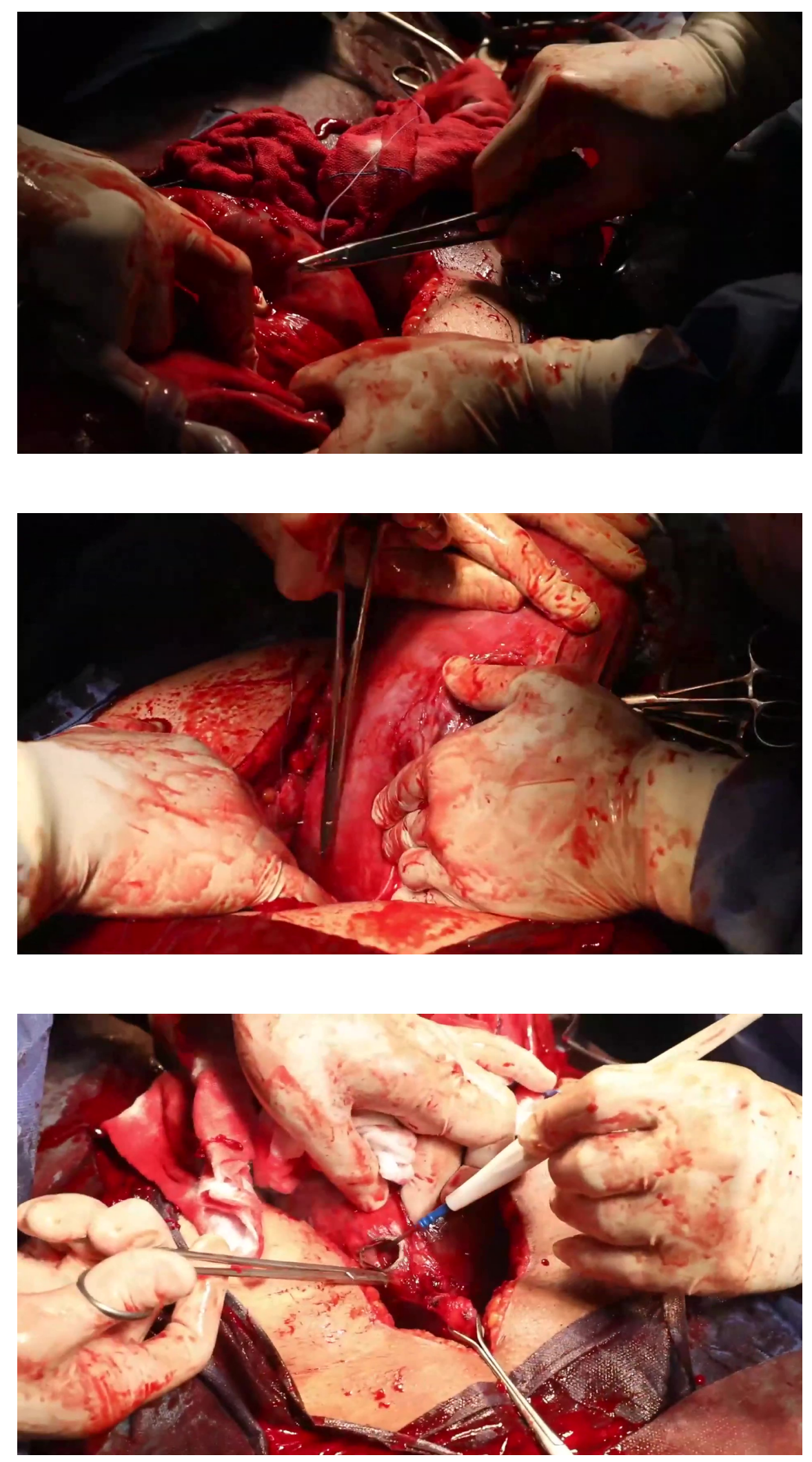

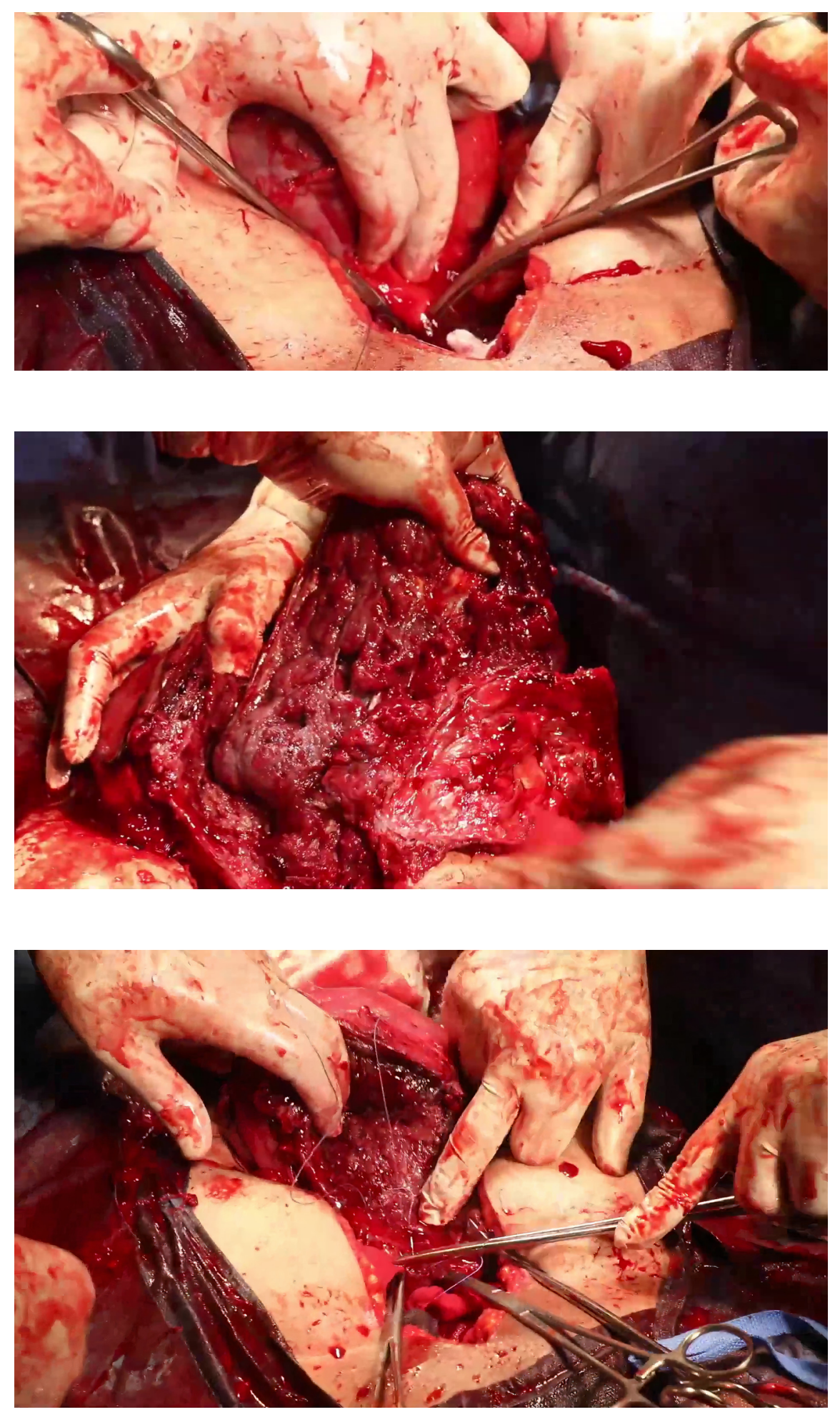

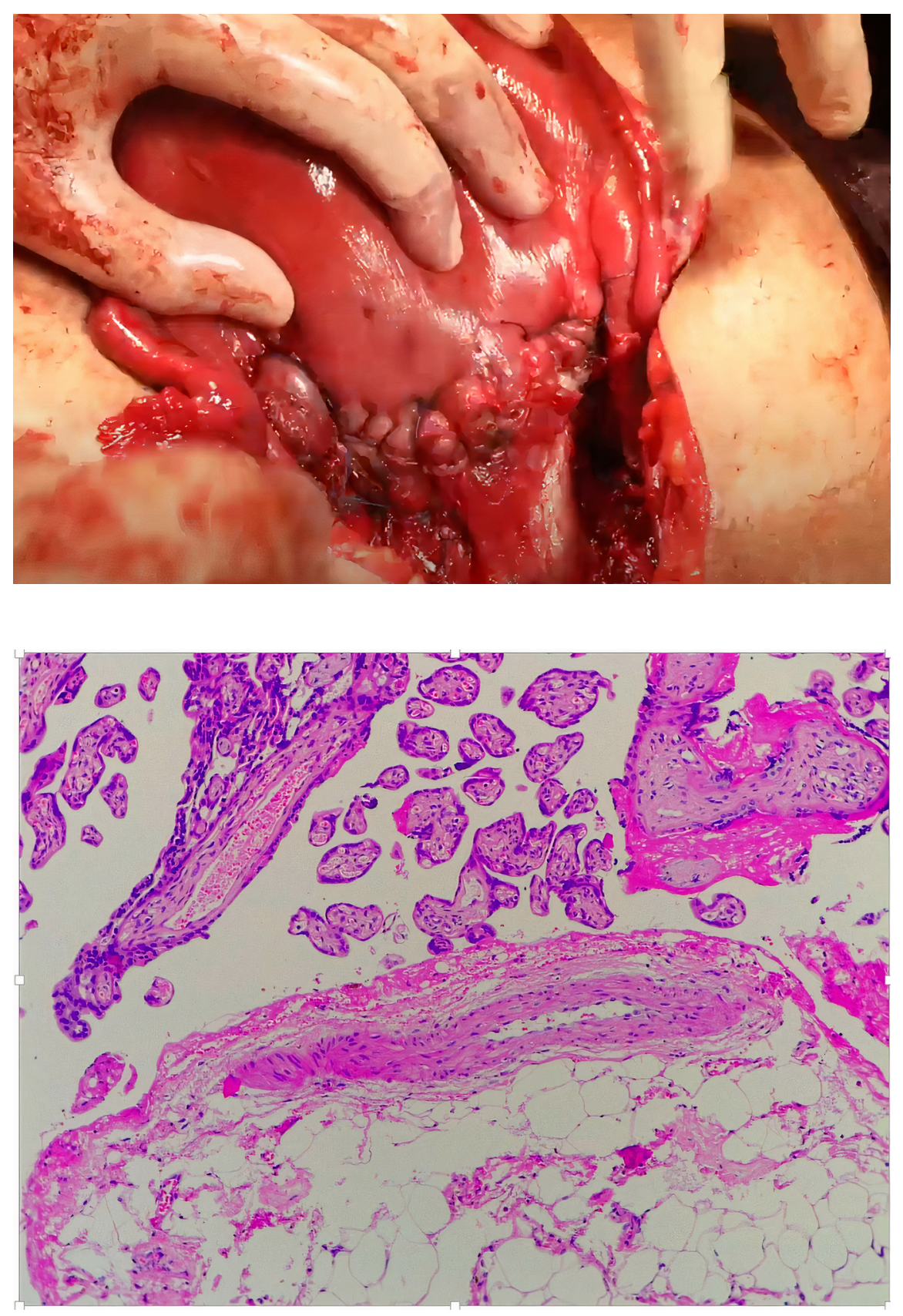


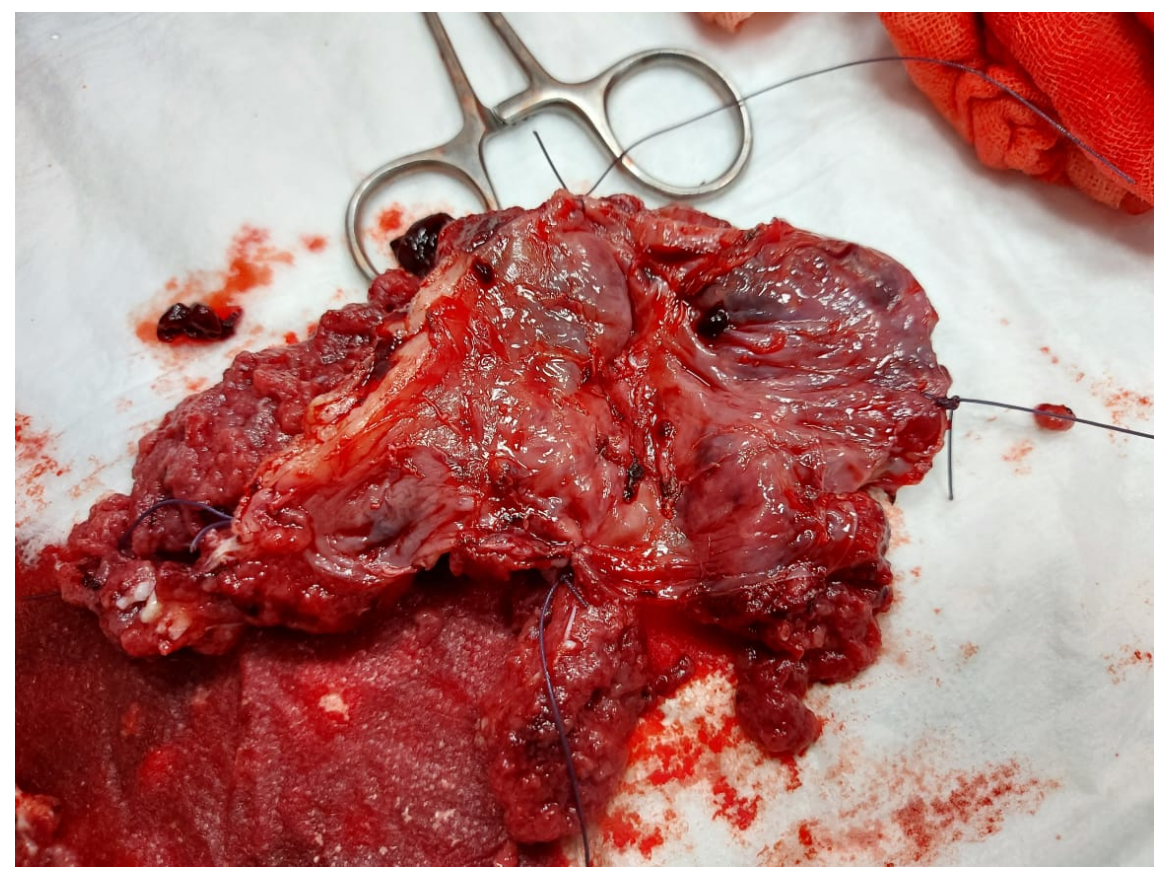

\section{Hosted file}

Figure 6.pdf available at https://authorea.com/users/367366/articles/486827-kasr-alainysimplified-conservative-uterine-surgery-for-abnormally-invasive-placenta-aip-surgicaltechnique

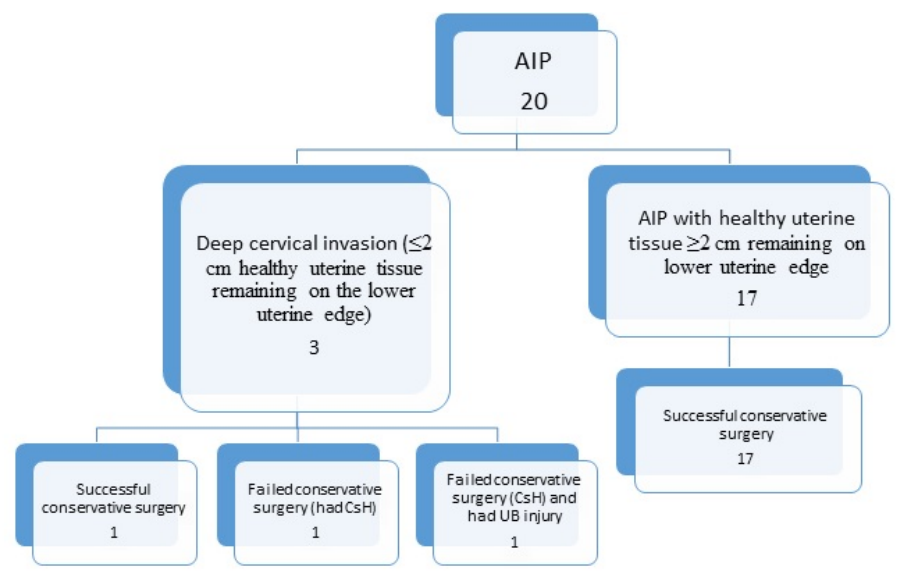

\section{Hosted file}

Table 1.pdf available at https://authorea.com/users/367366/articles/486827-kasr-alainysimplified-conservative-uterine-surgery-for-abnormally-invasive-placenta-aip-surgicaltechnique

\section{Hosted file}


Table 2.pdf available at https://authorea.com/users/367366/articles/486827-kasr-alainysimplified-conservative-uterine-surgery-for-abnormally-invasive-placenta-aip-surgicaltechnique

\section{Hosted file}

Table 3.pdf available at https://authorea.com/users/367366/articles/486827-kasr-alainysimplified-conservative-uterine-surgery-for-abnormally-invasive-placenta-aip-surgicaltechnique 